\title{
Resilience Informatics for Cyber-augmented Manufacturing Networks (CMN): Centrality, Flow and Disruption
}

\author{
Win P. V. NGUYEN, Shimon Y. NOF* \\ PRISM Center \& School of Industrial Engineering, Purdue University, USA \\ nguyen41@purdue.edu, nof@purdue.edu (*Corresponding author)
}

\begin{abstract}
Disruptive events during the past decade have inspired greater interest into the concept of resilience in manufacturing networks. In highly complex and interconnected manufacturing networks, disruptions are not isolated events, and the impacts in one location can propagate and affect other parts of the networks. Although the resilience concept in manufacturing networks is becoming better understood, there is a need for informatics and applications of informatics for the purpose of resilience in manufacturing networks. In this work, the Cyber-augmented Manufacturing Network (CMN) model is introduced along with three informatics developed for the purpose of understanding resilience. The CMN model captures the main components of a manufacturing network under disruption and their relationships. The three informatics and their eight indices provide important insights into the manufacturing networks under disruption. The model is validated with a case study with disruption repair to illustrate one possible application of the informatics.
\end{abstract}

Keywords: Resilient manufacturing, Manufacturing network, Manufacturing informatics, Cyber-physical systems.

\section{Introduction and Background}

Disruptive events during the past decade have inspired greater interest into the concept of resilience in cyber-physical systems (CPS): supply networks, manufacturing networks (MN) computer networks, transportation networks, and utility networks (Crucitti, Latora \& Marchiori, 2004). Externally, natural disasters and security concerns can disrupt the supply of certain raw materials, and intermediate production; damage or destroy the infrastructure, which can negatively impact manufacturing and production activities. Internally, the manufacturing equipment are subjected to wear and tear, breakdowns, malfunctions, and human errors, and require maintenance and repair to ensure productivity and quality (Sajadi, Esfahani \& Sorensen, 2011). This is particularly important in highly advanced and flexible manufacturing equipment, involving complex machinery and control. While a company may have limited controls and knowledge over external disruptions, the company can proactively manage internal disruptions to sustain resilient manufacturing operations.

Due to the complex interactions and interdependencies of a $\mathrm{MN}$, disruptions in one part of the $\mathrm{MN}$ can propagate and negatively hamper the production capacity of other parts of the $\mathrm{MN}$, affecting the performance and viability of the components of the entire system. Disruptions in MNs can negatively affect preceding and succeeding nodes, due to unfulfilled demands and/or supplies (Day, 2014; Gong, Mitchell, Krishnamurthy \& Wallace, 2014). Management and coordination of maintenance and repair in complex manufacturing networks typically faces the challenges of diversified machinery and limited personnel. As manufacturing equipment can be highly diverse: different machine types, different models, different brands, etc., especially for small and medium manufacturing businesses, where capital investments, knowledge, and business relationships are limited. Therefore, effective coordination and scheduling of repair and maintenance are required to minimize loss of system performance and recovery time. The importance of effective operations protocols is highlighted in tightly dependent manufacturing networks, where disruptions in essential machines could drastically hamper productivity of the entire network.

The literature on manufacturing systems has become increasingly focused on modelling manufacturing systems as networks of machines, or manufacturing networks (MN) (Huang, Cheng, \& Holt, 2007; Huang et al., 2007; Venkateshan, Mathur \& Ballou, 2008; Firmansyah \& Amer, 2013; Li, Mo \& Liu, 2013). In a MN, machines are represented as nodes, and the relationships between machines, or edges, are typically modelled as network flows, although other representations are possible. This approach enables the application of the knowledge from various network-related disciplines: network science, network theory, network flows. This approach also enables relatively more complex analysis to be applied and performed, such as dependency 
analysis and centrality analysis. While the increasing connectivity of CPSs enables greater levels of quality of service and customization, the increasing connectivity also brings about the important challenge of having to address security, resilience, and effective response, to ensure the performance and viability of the CPSs concerned.

Resilience is a concept derived from the field of ecology, and is increasingly becoming popular in engineering disciplines, particularly in research involving complex networks and systems (Reyes-Levalle \& Nof, 2015a, 2015b; Yodo \& Wang, 2016; Reyes-Levalle \& Nof, 2017; ReyesLevalle, 2018). The research surrounding $M N$ has made significant progress towards understanding the dependencies between nodes, system performances, complex causal relationship (Zhong $\&$ Nof, 2015; Fioriti \& Chinnici, 2017). Despite this progress, few researchers have investigated the aspect of resilience informatics and their possible applications in MNs. Researchers that investigate resilience and MNs typically look at the MNs from a supply chain/network management perspective (Topal \& Sahin, 2018), and rarely discuss disruptions requiring maintenance and repair. Other works discuss MNs and resilience measures, but not repair operations (Gao, Zhu \& Song, 2014; Gu, Jin \& Ni, 2014; Guariniello $\&$ DeLaurentis, 2017). Agility and resilience with MNs are also discussed from a supply chain management perspective (Ismail, Poolton \& Sharifi, 2011; Reyes-Levalle \& Nof, 2015a, 2015b; Thomas et al., 2015; Golini, Deflorin \& Scherrer, 2016; Seok, Kim \& Nof, 2016; ReyesLevalle \& Nof, 2017). This gap is addressed by our research, by the introduction of the Cyberaugmented Manufacturing Networks (CMN) model and the use of resilience informatics.

The CMN model and its informatics extend our previous research on the Dynamic Lines of Collaboration (DLOC) (Zhong \& Nof, 2015), and is developed based on the principle of emergent lines of collaboration and command, as well as e-Measure, as specified by the Collaborative Control Theory (CCT) (Nof, 2007). Both CCT and DLOC are methodologies developed and validated by Purdue PRISM Center, and are designed for engineering complex networks and cyber-physical systems. The DLOC model and protocols tackle disruptions effectively by performing the pathbased betweenness centrality analysis, and allocating repair agents to the nodes that have higher centrality measures.

The new CMN model is accompanied by three informatics: centrality, flow, and disruption, and their eight indices to support resilience. Building upon DLOC, the CMN model expands the centrality analysis to a trail-based centrality informatic that consists of four centrality indices. The trail-based centrality indices are considered more appropriate for directed and weighted manufacturing networks, as opposed to traditional centrality measures that are considered more appropriate for path-based networks. Furthermore, the CMN model introduces the flow informatic that consists of the flow dependency and influence indices, as well as the disruption informatic that consists of the disruption vulnerability and impact indices. Due to its advanced measures, indices, and protocols, the $\mathrm{CMN}$ model and its informatics significantly relies on the cyber aspects of the manufacturing networks, particularly communications and information processing. The CMN model is then applied (below) to a case study of a manufacturing network with rework capabilities, and the three developed informatics are applied and computed. One experiment with a team of repair agents is conducted to illustrate one possible application of the developed informatics and indices. The results show that the online scheduling protocols based on the CMN informatics outperform the traditional approach of selecting the most or the least critical failure first.

\section{Methodology}

In this section, the CMN model is presented (Figure 1). The CMN model has two components: the manufacturing network and the disruptions. The manufacturing network consists of machines (nodes) and flows (directed and weighted edges) between the machines. The direction of the flow indicates the supply direction, and the weight of the flow indicates the amount of supply from one machine to another. This network is subjected to a number of initial disruptions that affect the operational capabilities of several machines, thus reducing their output flows. The fully functional machines with reduced input flows will also have reduced output flows. The reduced output flow effect is propagated in the machines network downstream, ultimately causing inferior performance of the network. 


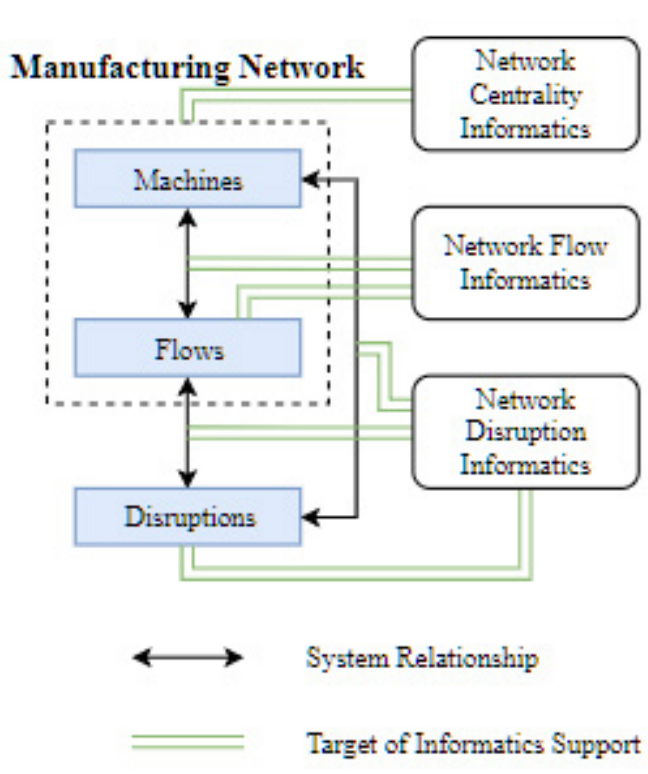

Figure 1. The CMN model

The entities and attributes of the CMN model are given in Table 1.

Table 1. The entities and attributes of the CMN model

\begin{tabular}{|l|l|}
\hline Type & Notation \\
\hline $\begin{array}{l}\text { The following entities are defined for the } \\
\text { CMN model: }\end{array}$ & $\begin{array}{l}C M R=(N L, E L, D N L) \\
\text { The manufacturing network concerned. }\end{array}$ \\
\hline Input & $\begin{array}{l}N L=\left\{n_{0}, n_{1} \ldots\right\} \\
\text { The set of machines (alternatively } \\
\text { called nodes). }\end{array}$ \\
\hline Input & $\begin{array}{l}E L=\left\{e_{0}, e_{1} \ldots\right\} \\
\text { The set of directed and weighted flows } \\
\text { (alternatively called edges). }\end{array}$ \\
\hline Input & $\begin{array}{l}D N L \subset N L \\
\text { The set of disrupted machines. }\end{array}$ \\
\hline Input & $\begin{array}{l}\text { The following attributes are defined for each } \\
\text { machine } \boldsymbol{n} \in \boldsymbol{N} \boldsymbol{L}\end{array}$ \\
\hline $\begin{array}{l}\text { Time- } \\
\text { based }\end{array}$ & $\begin{array}{l}\text { OS }(t, n) \in[0,1] \\
\text { The operational status of machine } n \text { at } \\
\text { time } t .0 / 1 \text { means the machine is not } \\
\text { producing/ producing at full capacity. }\end{array}$ \\
\hline $\begin{array}{l}\text { Time- } \\
\text { based }\end{array}$ & $\begin{array}{l}S S(t, n) \in[0,1] \\
\text { at supply status of machine } m\end{array}$ \\
\hline
\end{tabular}

\begin{tabular}{|c|c|}
\hline Derived & $\begin{array}{l}\operatorname{IEL}(n) \subset N L \\
\text { The set of input flows of machine } n \\
\operatorname{IEL}(n)=\left\{\left(n_{i}, n\right) \mid n_{i} \in N L\right\} .\end{array}$ \\
\hline Derived & $\begin{array}{l}O E L(n) \subset N L \\
\text { The set of output flows of machine } n \\
O E L(n)=\left\{\left(n, n_{j}\right) \mid n_{j} \in N L\right\} .\end{array}$ \\
\hline \multicolumn{2}{|c|}{$\begin{array}{l}\text { The following attributes are defined for each } \\
\text { flow } e=\left(n_{i}, n_{j}\right) \in E L\end{array}$} \\
\hline Derived & $\begin{array}{l}n_{i} \equiv N I(e), n_{j} \equiv N J(e) \in N L \\
n_{i} \text { denotes the input machine of flow } \\
e, \text { and } n_{i} \text { denotes the output machine } \\
\text { of flow } e .\end{array}$ \\
\hline Input & $\begin{array}{l}S R(0, e) \in \mathbb{R}^{+} \\
\text {The given initial (and maximum) } \\
\text { supply rate of this flow. }\end{array}$ \\
\hline & $\begin{array}{l}S R(t, e) \in[0, S R(0, e)] \\
\text { The supply rate of this flow at time } \\
t . \text { For example, } S R(2, e)=3 \text { and } \\
e=\left(n_{0}, n_{2}\right) \Leftrightarrow \text { at time } t=2, n_{0} \\
\text { supplies } n_{2} \text { with } 3 \text { units of flow. }\end{array}$ \\
\hline \multicolumn{2}{|c|}{ The disruptions are defined as follows: } \\
\hline Input & $\begin{array}{l}D N L \in N L \\
\text { The set of disrupted machines. }\end{array}$ \\
\hline \multicolumn{2}{|c|}{ For each disrupted node $n_{d} \in D N L$ : } \\
\hline Input & $\begin{array}{l}O S\left(0, n_{d}\right) \in[0,1) \\
\text { This machine is disrupted at time } t=0 \\
\text { and the value is given by the problem. }\end{array}$ \\
\hline \multicolumn{2}{|c|}{$\begin{array}{l}\text { All other machines function normally. } \\
O S(0, n)=1, \forall n \in N L-D N L \\
\text { And all machines are supplied initially. } \\
S S(0, n)=1, \forall n \in N L\end{array}$} \\
\hline
\end{tabular}

The network performance metric is defined as follows:

$$
N P(t)=\frac{\sum_{n}^{N_{\text {sink }}} S S(t, n)}{\left|N_{\operatorname{sink}}\right|}
$$

with $N_{\text {sink }}=\{n \in N L \mid O E L(n) \equiv \varnothing\}$.

The expression is the average of the supply status of all sink nodes (machines that are output nodes of the manufacturing network). 
The time-based system relationships include:

$\forall n \in N L \mid \operatorname{IEL}(n) \neq \varnothing$,

$S S(t, \mathrm{n})=\frac{\sum_{e \in I E L(N)} S R(t-1, \mathrm{e})}{\sum_{e \in I E L(N)} S R(0, \mathrm{e})}$

$\forall n \in N L \mid \operatorname{IEL}(n) \equiv \varnothing, S S(t, \mathrm{n})=O S(0, n)$

$S R(t, e)=S R(0, e)$

$\times \min \{O S(0, N I(e)), S S(t, N I(e))\}$

Equation (2a) applies to the non-source machines, and describes the relationship between a machine and its input flows. Equation (2b) applies to the source machines (machines with no input flows). Equation (3) describes the relationship between a flow and its input machine.

Three informatics are developed for the purpose of understanding the resilience of the manufacturing network. The informatics provide each machine with a set of indices, with each index indicating the degree of importance of a machine with respect to the objective of the index concerned. The informatics and their indices are listed in Table 2.

Table 2. Informatics and their indices

\begin{tabular}{|l|l|l|}
\hline Informatic & $\begin{array}{l}\text { Index } \\
\text { code }\end{array}$ & Index name \\
\hline $\begin{array}{l}\text { Network } \\
\text { centrality }\end{array}$ & $C I_{0}$ & Downstream centrality \\
\cline { 2 - 3 } & $C_{1}$ & Upstream centrality \\
\cline { 2 - 3 } & $C I_{2}$ & $\begin{array}{l}\text { Influence-from-source } \\
\text { centrality }\end{array}$ \\
\cline { 2 - 3 } & $C_{3}$ & $\begin{array}{l}\text { Influence-to-sink } \\
\text { centrality }\end{array}$ \\
\hline $\begin{array}{l}\text { Network } \\
\text { flow }\end{array}$ & $F D I$ & Flow dependency \\
\cline { 2 - 3 } & $F I I$ & Flow influence \\
\hline $\begin{array}{l}\text { Network } \\
\text { disruption }\end{array}$ & $D V I$ & Disruption vulnerability \\
\hline & $D I I$ & Disruption impact \\
\hline
\end{tabular}

The network centrality informatics analyses the network structure and provides four centrality indices for each machine, indicating its relative importance compared to other machines. The computation is as follows.

Enumerate all possible directed trails of the network. A directed trail is defined as a directed walk without any repetition of edges. Directed trails are used instead of directed paths because the manufacturing network could be defined with rework and self-loops, and directed cycles would exist. In the case with rework, directed paths (which do not allow repetition of nodes) will not fully reflect the complex relationships between the nodes. The supply rate of each edge $e=\left(n_{i}, n_{j}\right)$ is normalized into:

$\operatorname{NSR}\left(\left(n_{i}, n_{j}\right)\right)=\frac{S R\left(0,\left(n_{i}, n_{j}\right)\right)}{\sum_{e_{i} \in \operatorname{IEL}\left(n_{j}\right)} S R\left(0, e_{j}\right)}$

A trail $T=\left(n_{i} \ldots n_{j}\right)=\left(e_{a}, e_{b} \ldots\right)$ with $|T|$ as the number of edges of the trail, would then carry a value of

$C I(T)=\frac{\sum_{e}^{T} N S R(e)}{|T|}$

Then the measures $C I_{0}(n), C I_{1}(n), C I_{2}(n)$, $C_{3}(n)$ of the node $n$ are defined as:

$$
\begin{aligned}
& C I_{0}(n)=\sum_{T=\left(n_{i}=n \ldots n_{j}\right)} C I(T) \\
& C I_{1}(n)=\sum_{T=\left(n_{i} \ldots n_{j} \equiv n\right)} C I(T) \\
& C I_{2}(n)=\sum_{T=\left(n_{i} \ldots n_{j} \equiv n\right) \mid I E L\left(n_{i}\right)=\varnothing} C I(T) \\
& C I_{3}(n)=\sum_{T=\left(n_{i}=n \ldots n_{j}\right) \mid O E L\left(n_{j}\right)=\varnothing} C I(T)
\end{aligned}
$$

The downstream centrality index $C I_{0}(n)$ is the summation of the $C I(T)$ values of all trails $T$ that originate from node $n$. The index $C I_{0}(n)$ indicates the impact of node $n$ on the other nodes of the network. The upstream centrality index $C I_{1}(n)$ is the summation of the $C I(T)$ values of all trails $T$ that end at node $n$. The index $C I_{1}(n)$ indicates the dependency of node $n$ on the other nodes of the network. The influence-from-source centrality index $\mathrm{CI}_{2}(n)$ is the summation of the $C I(T)$ values of all trails that originate from a source node (a node without input edges) and end at node $n$. The index $\mathrm{CI}_{2}(n)$ indicates the dependency of node $n$ from the source nodes of the network. The influence-to-sink centrality index $\mathrm{CI}_{3}(n)$ is the summation of the $C I(T)$ values of all trails that originate from node $n$ and end at a sink node (a node without output edges). 
The index $\mathrm{CI}_{3}(n)$ indicates the impact of node $n$ on the sink nodes of the network.

The network flow informatics analyses the flows and the machine-flow relationship to provide two flow indices. The two flow indices $F I I(n)$ and $F D I(n)$ are computed as follows.

Call $F I\left(n_{i}, n_{j}\right)$ the steady-state supply status of node $n_{j}$ given that node $n_{i}$ is fully supplied initially and all other nodes are not fully supplied initially, and all source nodes are disabled.

$F I\left(n_{i}, n_{j}\right)=S S\left(+\infty, n_{j}\right)$

with $O S\left(t, n_{i}\right)=S S\left(t, n_{i}\right)=1, \forall t \in[0,+\infty)$ and $\operatorname{OS}\left(0, n^{*}\right)=1, S S\left(0, n^{*}\right)=0, \forall n^{*} \neq n_{i} \in N L \mid \operatorname{IEL}\left(n^{*}\right) \neq \varnothing$ and

$\operatorname{OS}\left(0, n_{s}^{*}\right)=\operatorname{SS}\left(0, n_{s}^{*}\right)=0, \forall n^{*} \neq n_{i} \in N L \mid \operatorname{IEL}\left(n^{*}\right) \equiv \varnothing$ and equations (2a), (2b), and (3) hold.

Then, the flow influence index $F I I(n)$ is computed as follows.

$F I I(n)=\sum_{n^{*} \neq n}^{N L} \frac{F I\left(n, n^{*}\right)}{|N L|-1}$

And the flow dependency index $F D I(n)$ is computed as follows.

$F D I(n)=\sum_{n^{*} \neq n}^{N L} \frac{F I\left(n^{*}, n\right)}{|N L|-1}$

The flow influence index $F I I(n)$ indicates how much node $n$ influences the supply status of all other nodes. The flow dependency index $F D I(n)$ indicates how much the supply status of node $n$ is dependent on all other nodes' supply statuses.

The network disruption informatics analyses the disruptions, disruption-flow, and disruptionmachine relationship to provide two disruption indices. The two disruption indices $\operatorname{DII}(n)$ and $\operatorname{DVI}(n)$ are computed as follows.

Call $D I\left(n_{i}, n_{j}\right)$ the steady-state supply status of node $n_{i}$ given that node $n_{i}$ is fully disrupted and all other nodes are fully supplied and operational.

$D I\left(n_{i}, n_{j}\right)=S S\left(+\infty, n_{j}\right)$

with $O S\left(t, n_{i}\right)=S S\left(t, n_{i}\right)=0, \forall t \in[0,+\infty)$ and $O S\left(0, n^{*}\right)=1, S S\left(0, n^{*}\right)=1, \forall n^{*} \neq n_{i} \in N L$.
Then, with $N L_{\text {sink }}$ as the set of sink nodes, the disruption impact index $\operatorname{DII}(n)$ is computed as follows.

$D I I(n)=1-\sum_{n^{*} \neq n}^{N L_{\text {sink }}} \frac{D I\left(n, n^{*}\right)}{|N L|-1}$

The disruption vulnerability index $\operatorname{DVI}(n)$ is computed as follows.

$D V I(n)=\max _{n^{*} \in N L_{\text {source }}-\{n\}}\left(1-D I\left(n^{*}, n\right)\right)$

The disruption impact index $\operatorname{DII}(n)$ indicates how much a disruption from node $n$ impacts the total supply status of the sink nodes, which is the network performance. The disruption vulnerability index $\operatorname{DVI}(n)$ indicates the worst disruption's impact on the supply status of node $n$.

\section{Experiments - Informatics and Disruption Repair based on Informatics}

In this section, the CMN model is validated with numerical experiments, and one application of the CMN model's informatics is illustrated. The selected manufacturing network has 20 nodes and rework capability (Figure 2).

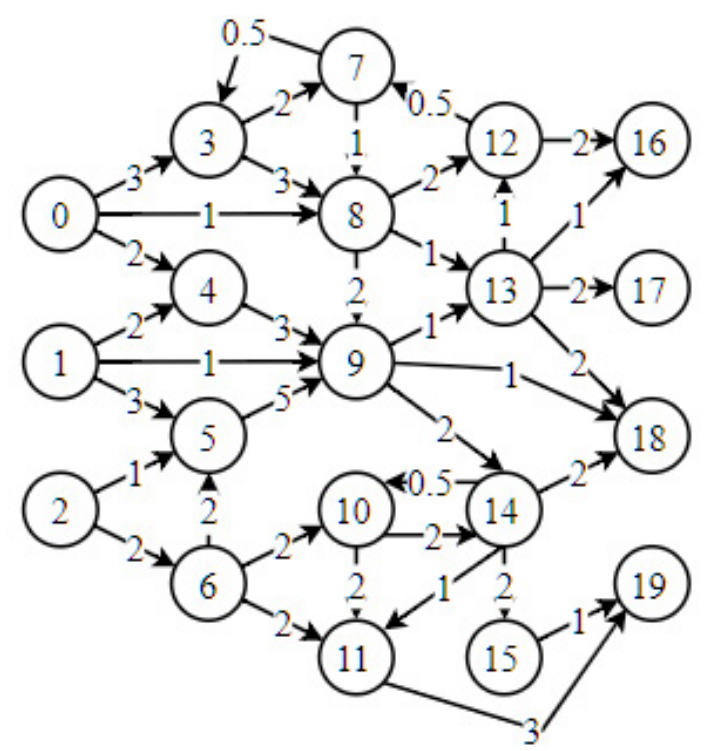

Figure 2. Manufacturing Network with Rework

Based on this network, the indices are computed and given in Table 3 (with CI0, CI1, CI2, and CI3 normalized). 
Table 3. Experiment Results - Indices

\begin{tabular}{|c|c|c|c|c|c|c|c|c|}
\hline $\mathbf{n}$ & $\mathbf{C I 0}$ & $\mathbf{C I 1}$ & $\mathbf{C I 2}$ & $\mathbf{C I 3}$ & FII & FDI & DII & DVI \\
\hline $\mathbf{0}$ & 1 & 0 & 0 & 1 & .4 & 0 & .48 & 0 \\
\hline $\mathbf{1}$ & .4 & 0 & 0 & .38 & .17 & 0 & .19 & 0 \\
\hline $\mathbf{2}$ & .33 & 0 & 0 & .3 & .32 & 0 & .33 & 0 \\
\hline $\mathbf{3}$ & .52 & .04 & .04 & .53 & .24 & .06 & .34 & 1 \\
\hline $\mathbf{4}$ & .13 & .01 & .01 & .12 & .07 & .05 & .11 & .5 \\
\hline $\mathbf{5}$ & .14 & .01 & .01 & .13 & .11 & .07 & .19 & .5 \\
\hline $\mathbf{6}$ & .17 & .01 & .01 & .15 & .24 & .05 & .29 & 1. \\
\hline $\mathbf{7}$ & .3 & .05 & .06 & .3 & .08 & .13 & .12 & .97 \\
\hline $\mathbf{8}$ & .3 & .09 & .09 & .31 & .2 & .11 & .43 & .99 \\
\hline $\mathbf{9}$ & .13 & .11 & .1 & .13 & .2 & .12 & .42 & .46 \\
\hline $\mathbf{1 0}$ & .02 & .17 & .18 & .02 & .12 & .12 & .18 & .91 \\
\hline $\mathbf{1 1}$ & 0 & .52 & .53 & .01 & .04 & .16 & .19 & .88 \\
\hline $\mathbf{1 2}$ & .13 & .1 & .09 & .13 & .06 & .18 & .19 & .88 \\
\hline $\mathbf{1 3}$ & .12 & .15 & .14 & .13 & .13 & .17 & .49 & .65 \\
\hline $\mathbf{1 4}$ & .02 & .18 & .19 & .02 & .12 & .17 & .22 & .57 \\
\hline $\mathbf{1 5}$ & 0 & .44 & .43 & 0 & .01 & .22 & .06 & .57 \\
\hline $\mathbf{1 6}$ & 0 & .63 & .59 & 0 & 0 & .23 & 0 & .8 \\
\hline $\mathbf{1 7}$ & 0 & .35 & .33 & 0 & 0 & .22 & 0 & .65 \\
\hline $\mathbf{1 8}$ & 0 & .79 & .79 & 0 & 0 & .21 & 0 & .4 \\
\hline $\mathbf{1 9}$ & 0 & 1 & 1 & 0 & 0 & .23 & 0 & .8 \\
\hline
\end{tabular}

One useful application of the three informatics in resilient manufacturing is the timely allocation of repair resources to repair disruptions. In this case, a collaborative team of five repair agents is given (Figure 3). The weights of the agents indicate the invidual operational status recovery amount. The weights of the connections between agents indicate the additional recovery amount if two agents cooperate to repair a machine.

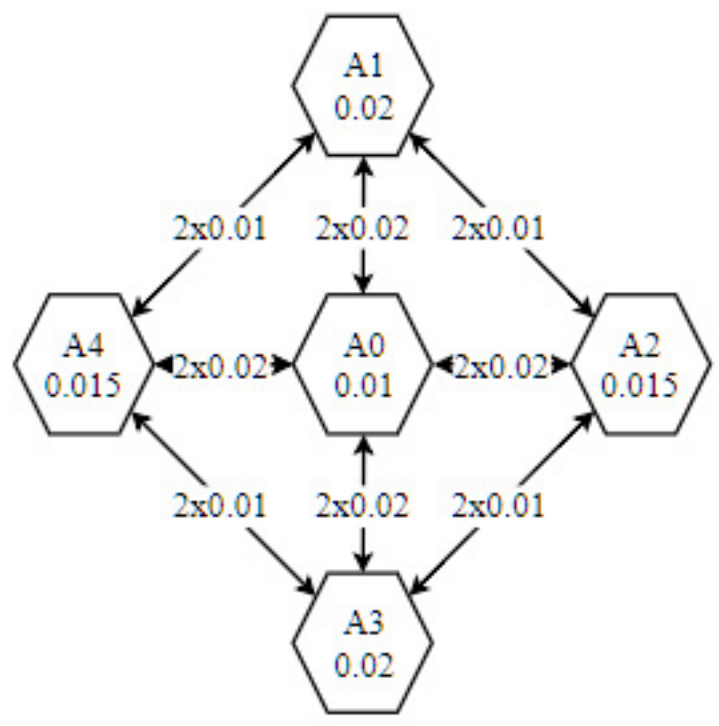

Figure 3. Agent Network
The case study is simulated with six different failure scenarios, with the number of disruptions/ failures ranging from five to ten, and each scenario is simulated 20 times. The nodes subjected to initial disruptions are randomly selected, with each node having the same probability of being selected. With this case study, two performance measures are used to evaluate the performance with the informatics. The first measure, recovery time, is the time $t$ when the simulation ends. The second measure, total lost performance, is calculated as $\sum(1-N P(t))$. A total of eighteen different priority rules (protocols) are applied for the repair agents. Two traditional priority rules include the traditional most-critical-failure-first (MCFF) and least-critical-failure-first (LCFF). The other sixteen rules are based on the given informatics' indices. The results are presented in Figure 4. The results indicate that the use of the developed indices can support making better, timely repair decisions for the agent network.

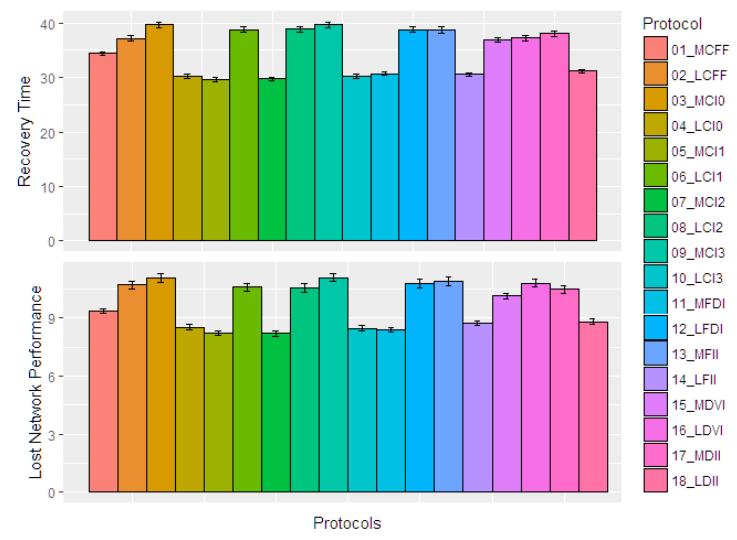

Figure 4. Experiment Results - Repair

\section{Conclusions and Future Works}

In this work, the new CMN model and three informatics are introduced to address the need for modelling and supporting resilience for manufacturing networks. This work also addresses the need for informatics and application of informatics in the context of resilience for manufacturing networks. The CMN model provides a framework for modelling cyberaugmented manufacturing networks and the effects of disruptions on the network. The informatics and indices provided by the $\mathrm{CMN}$ model have multiple possible applications, and in the work presented in 
this article, are used to support disruption repair activities allocation and scheduling.

The results of the experiments with repair agents indicate that the CMN model's informatics developed could be applied and useful towards the following purposes:

1. MN control, management. and decision support.

2. MN design and redesign to prepare against disruptions.

3. Disruption preparedness by supply backup and inventory control.

4. Disruption response by repair, emergency outsourcing, and flow rerouting.

\section{REFERENCES}

1. Crucitti, P., Latora, V. \& Marchiori, M. (2004). Model for cascading failures in complex networks, Phys Rev E Stat Nonlin Soft Matter Phys, 69(4 Pt 2), 045104. doi:10.1103/PhysRevE.69.045104

2. Day, J. M. (2014). Fostering emergent resilience: the complex adaptive supply network of disaster relief, International Journal of Production Research, 52(7), 19701988. doi:10.1080/00207543.2013.787496

3. Fioriti, V. \& Chinnici, M. (2017). Node Seniority Ranking in Networks, Studies in Informatics and Control, 26(4), 397-402. doi:10.24846/v26i4y201703

4. Firmansyah, M. R. \& Amer, Y. (2013). A review of collaborative manufacturing network models, Int. J. Mater. Mech. Manufact, 1(1), 6-12.

5. Gao, Z., Zhu, Q. \& Song, T. (2014). Analysis and research on dynamic models of complex manufacturing network cascading failures. In 2014 Sixth International Conference on Intelligent Human-Machine Systems and Cybernetics (pp. 388-391).

6. Golini, R., Deflorin, P. \& Scherrer, M. (2016). Exploiting the potential of manufacturing network embeddedness: An OM perspective, International Journal of Operations \& Production Management, 36(12), 1741-1768.

7. Gong, J., Mitchell, J. E., Krishnamurthy, A. \& Wallace, W. A. (2014). An interdependent
This research could be further expanded into several challenging directions:

1. MN design and redesign based on the support of resilience informatics.

2. Frameworks and principles of $\mathrm{MN}$ resilience control and management.

3. Validation of resilience informatics in more general MN models.

\section{Acknowledgement}

This research has been developed with partial support from the Production, Robotics, and Integration Software for Manufacturing \& Management (PRISM) Center at Purdue University.

layered network model for a resilient supply chain, Omega, 46, 104-116. doi:10.1016/j. omega.2013.08.002

8. Gu, X., Jin, X. \& Ni, J. (2014). Resilience Measures of Manufacturing Systems under Disruptions. In ASME. International Manufacturing Science and Engineering Conference, Volume 1: Materials; Micro and Nano Technologies; Properties, Applications and Systems; Sustainable Manufacturing (pp. V001T04A007).

9. Guariniello, C. \& DeLaurentis, D. (2017). Supporting design via the System Operational Dependency Analysis methodology, Res Eng Design, 28, 53-69.

10. Huang, C.-Y., Cheng, K. \& Holt, A. (2007). An integrated manufacturing network management framework by using mobile agent, The International Journal of Advanced Manufacturing Technology, 32(7), 822-833. doi:10.1007/s00170-005-0378-1

11. Huang, C.-Y., Holt, A., Monk, J. \& Cheng, K. (2007). The application of dependency management in an integrated manufacturing network framework, The International Journal of Advanced Manufacturing Technology, 33(3-4), 354-364.

12. Ismail, H. S., Poolton, J. \& Sharifi, H. (2011). The role of agile strategic capabilities in achieving resilience in manufacturing-based small companies, International Journal of Production Research, 49(18), 5469-5487. 
13. Li, J., Mo, R. \& Liu, L. L. (2013). Analysis of service-oriented manufacturing network based on complex network. In Applied Mechanics and Materials (pp. 401-405).

14. Nof, S. Y. (2007). Collaborative control theory for e-Work, e-Production, and e-Service, Annual Reviews in Control, 31, 281-292.

15. Reyes-Levalle, R. (2018). Resilience by Teaming in Supply Chains and Networks. Springer International Publishing.

16. Reyes-Levalle, R. \& Nof, S. Y. (2015a). A resilience by teaming framework for collaborative supply networks, Computers \& Industrial Engineering, 90, 67-85.

17. Reyes-Levalle, R. \& Nof, S. Y. (2015b). Resilience by teaming in supply network formation and re-configuration, Int. J. Production Economics, 160, 80-93.

18. Reyes-Levalle, R. \& Nof, S. Y. (2017). Resilience in supply networks: Definition, dimensions, and levels, Annual Reviews in Control, 43, 224-236.

19. Sajadi, S. M., Esfahani, M. M. S. \& Sorensen, K. (2011). Production control in a failureprone manufacturing network using discrete event simulation and automated response surface methodology, International Journal of Advanced Manufacturing Technology, 53(1-4), 35-46. doi:10.1007/s00170-0102814-0
20. Seok, H., Kim, K. \& Nof, S. Y. (2016). Intelligent contingent multi-sourcing model for resilient supply networks, Expert Systems With Applications, 51, 107-119.

21. Thomas, A., Pham, D. T., Francis, M. \& Fisher, R. (2015). Creating resilient and sustainable manufacturing businesses - a conceptual fitness model, International Journal of Production Research, 53(13), 3934-3946.

22. Topal, B. \& Sahin, H. (2018). The Influence of Information Sharing in the Supply Chain Process on Business Performance: An Empirical Study, Studies in Informatics and Control, 27(2), 201-212.

23. Venkateshan, P., Mathur, K. \& Ballou, R. H. (2008). An efficient generalized networksimplex-based algorithm for manufacturing network flows, Journal of Combinatorial Optimization, 15(4), 315-341. doi:10.1007/ s10878-007-9080-6

24. Yodo, N. \& Wang, P. (2016). Engineering resilience quantification and system design implications: a literature survey, Journal of Mechanical Design, 138(11), 111408.

25. Zhong, H. \& Nof, S. Y. (2015). The dynamic lines of collaboration model: collaborative disruption response in cyberphysical systems, Computers \& Industrial Engineering, 87, 370-382. doi:doi. org/10.1016/j.cie.2015.05.019 